\title{
Impacto en la morbimortalidad por COVID-19 según las regulaciones del uso de mascarilla
}

\author{
Marlon E. Saavedra-Delgado, ${ }^{1 *}$ Ana Villaseñor-Todd, ${ }^{2}$ Sandra P. Caicedo-Agudelo, ${ }^{3}$ \\ David A. Lázaro-Presenda y Bernardo Ng-Solís ${ }^{2}$ \\ ${ }^{1}$ Asociación Psiquiátrica Mexicana, Ciudad de México; ${ }^{2}$ Hospital Regional de Monterrey, Instituto de Seguridad y Servicios Sociales de los

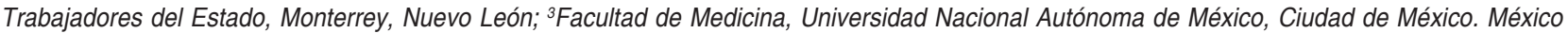

\section{Resumen}

Introducción: Actualmente, la mascarilla representa un símbolo incomparable cuyo valor pasó del trabajo clínico a impactar en la contención de la propagación del virus SARS-CoV-2; se convirtió en un elemento de discordia en la población general. Objetivo: Establecer el impacto de las políticas de uso de mascarilla en la morbimortalidad por COVID-19. Métodos: Se asociaron las variables de política de uso de cubrebocas, obtenidas de la página web del movimiento científico \#Masks4All, con el número de contagios, muertes y aplanamiento de la curva publicados por el centro de recursos de la Universidad Johns Hopkins y EndCoronavirus.org. Resultados: Las políticas de uso de mascarilla fueron de tipo universal (requerido en tiendas, restaurantes, transporte público), parcial (recomendado, requerido en cualquier lugar público) y ausente. Las asociaciones de la política de uso de mascarilla con casos totales $(p=0.01)$, casos por millón $(p=0.04)$ y muertes por millón de habitantes $(p=0.02)$ resultaron estadísticamente significativas. Las asociaciones de las variables con la tendencia de la curva epidemiológica también resultaron estadísticamente significativas $(p=0.00)$. Conclusión: La recomendación del uso generalizado de mascarilla es una medida con suficiente respaldo científico para reducir el número de contagios y muertes por COVID-19.

PALABRAS CLAVE: COVID-19. SARS-CoV-2. Mascarilla. Cubrebocas. Morbimortalidad.

\section{Impact on COVID-19 morbidity and mortality according to the regulations on the use of face mask}

\section{Abstract}

Introduction: Currently, the face mask represents an incomparable symbol whose value went from clinical work to impacting the containment of the spread of SARS-CoV-2, although it has become an element of discord in the general population. Objective: To establish the impact of face mask use policies on COVID-19 morbidity and mortality. Methods: Face mask use policy variables, obtained from the website of the \#Masks4All scientific movement, were associated with the number of infections, deaths and flattening of the curve published by the Johns Hopkins University resource center and EndCoronavirus.org. Results: Face mask use policies were universal (required in shops, restaurants, public transport), partial (recommended, required in any public place) and absent. Associations of the face mask use policy with total cases $(p=0.01)$, cases per million $(p=0.04)$ and deaths per million population $(p=0.02)$ were statistically significant. Associations of the variables with the epidemiological curve trend were also statistically significant $(p=0.00)$. Conclusion: The recommendation for face mask widespread use is a measure with sufficient scientific support to reduce the number of COVID-19-related infections and deaths.

KEY WORDS: COVID-19. SARS-CoV-2. Face mask. Morbidity. Mortality.

Correspondencia:

*Marlon E. Saavedra-Delgado

E-mail: marlonsaavedradelgado@gmail.com

DOI: 10.24875/GMM.20000801
Gac Med Mex. 2021;157:288-295

Disponible en PubMed

www.gacetamedicademexico.com CC BY-NC-ND (http://creativecommons.org/licenses/by-nc-nd/4.0/). 


\section{Introducción}

Stanley Ipkiss ("La Máscara"): "Es (la máscara) un lazo de fuerza... Se supone que debe hacerte sentir poderoso". Este diálogo cinematográfico se adapta perfectamente al protagonismo de las mascarillas durante la actual pandemia, las cuales se han convertido en un sinónimo de autoridad, una posesión valiosa. ${ }^{1}$ El simbolismo psicológico de la máscara se explica por el uso de las expresiones faciales para construir ideas sobre sentimientos y deseos, por lo que la distorsión suscitada por la mascarilla fomenta una respuesta individual que funciona en dos modos: uno controlado y otro implícito. ${ }^{2}$

El uso de la mascarilla en el campo clínico se remonta a 1700, con la teoría miasmática, que popularizó las famosas máscaras de "médico de la plaga" o "doctores de pico", descritas por Charles de Lorme." En un capítulo de su teoría operativa, Martin Kirschner (1879-1942) se refirió a las mascarillas como "medidas para combatir infecciones". En la década de 1920, se utilizaron por primera vez en el campo quirúrgico en Alemania y Estados Unidos. ${ }^{3}$

El uso de equipo de protección contra infecciones respiratorias dentro del ámbito clínico no tiene cuestionamiento en relación con su utilidad. ${ }^{4,5}$ Esto ha permitido trasladar dicha medida a la población general, con un impacto benéfico, ${ }^{5}$ por lo que incluso mascarillas elaboradas con materiales caseros se consideran una mejor alternativa a la ausencia de protección. ${ }^{5,6}$ Las más comunes en población general están elaboradas con material quirúrgico de menor filtración, así como de tela de prendas de uso diario, ${ }^{7,8}$ con variaciones de filtración de 9 a $98 \%,{ }^{7}$ que disminuye con la reutilización y los ciclos de limpieza. ${ }^{8}$

Al inicio de la actual pandemia, la recomendación general respecto al uso de mascarillas era la de racionarlas para el ámbito médico, pues se consideraba que la evidencia no proveía información contundente para indicarla ampliamente; ${ }^{9} \sin$ embargo, la preocupación de la comunidad científica generó un debate que llevó a la difusión de múltiples publicaciones que soportaban su utilidad.10,11 Tras sopesar los datos científicos, la Organización Mundial de la Salud actualizó la pauta para el uso generalizado. ${ }^{12}$ Una revisión sistemática y metaanálisis que publicó Lancet en junio de 2020 fue el respaldo final a favor de la indicación. ${ }^{13} \mathrm{~A}$ pesar de lo descrito, no todos los países consideraron hacer de esta medida un elemento obligatorio para sus ciudadanos.
El objetivo de este estudio fue comparar el impacto de la dispersión del virus SARS-CoV-2 entre países con diferentes políticas de uso de mascarillas.

\section{Métodos}

Se diseñó un estudio observacional, descriptivo, transversal. La información recabada estuvo integrada por las cifras de casos y muertes por COVID-19 registrados en la página oficial del Centro de Recursos de Coronavirus de la Universidad Johns Hopkins (https://coronavirus.jhu.edu/map.html). El tipo de política frente al uso de mascarillas se obtuvo de la página oficial del movimiento \#Mask4All (https://masks4all.co). Se sumó una variable de aplanamiento de la curva, con datos obtenidos de la página oficial del movimiento ENDCORONAVIRUS.org (https://www. endcoronavirus.org/countries). La fecha para recabar los datos fue el 30 de agosto de 2020.

El análisis estadístico fue realizado según la prueba de normalidad de Kolmogorov-Smirnov; se consideró distribución normal en caso de $p>0.05$ (para aplicar prueba de KrusKal-Wallis), distribución no normal en caso de $p<0.05$ (para aplicar prueba de Anova), estableciendo un valor de $p<0.05$ como estadísticamente significativo. Para la relación entre la política de uso de mascarillas y el aplanamiento de la curva, se aplicó la prueba de chi cuadrada. Se utilizó el programa SPSS.

\section{Resultados}

Se recabaron datos de un total de 215 países; 129 países contaron con la información correspondiente para los análisis bivariados. Las categorías de la política del uso de cubrebocas fueron las siguientes: universal, $U(5.08 \%)$; requerido en tiendas, restaurantes, transporte público, TRT (29.94\%); parcial, P (11.30\%); recomendado, R (8.47\%); requerido en cualquier lugar público, LP (37.85\%) y ausente, A (7.34 \%). La clasificación de la curva epidemiológica se indicó de la siguiente forma: tendencia alta, cTA (62.41\%); tendencia a la baja, cTB (13.53\%); aplanada, cA (24.06\%). A nivel mundial, el número de casos y muertes totales correspondió a 25376529 y 850 140, respectivamente; y el de casos y muertes por millón, a 3256 y 109.1, respectivamente.

Los países con el mayor número de casos totales (Tabla 1) fueron Estados Unidos (6172 082, P), Brasil (3862 311, P), India (3619 169, U), Rusia (990 326, P), Perú (647 166, LP), Suráfrica (625 056, LP), Colombia 
Tabla 1. Los 10 países con mayor número de contagios al $\mathbf{3 0}$ agosto de 2020

\begin{tabular}{|l|c|c|c|c|c|}
\hline País & Total de casos & $\begin{array}{c}\text { Casos/1 } 000 \text { 000 } \\
\text { población }\end{array}$ & Muertes totales & $\begin{array}{c}\text { Muertes/ } 1000000 \\
\text { población }\end{array}$ & $\begin{array}{c}\text { Política de uso de } \\
\text { mascarilla }\end{array}$ \\
\hline Estados Unidos & 6172082 & 18629 & 187215 & 565 & P \\
\hline Brasil & 3862311 & 18149 & 120896 & 568 & P \\
\hline India & 3619169 & 2618 & 64617 & 47 & P \\
\hline Rusia & 990326 & 6786 & 17093 & 117 & LP \\
\hline Perú & 647166 & 19584 & 28788 & 871 & LP \\
\hline Sudáfrica & 625056 & 10518 & 14028 & 236 & TRT \\
\hline Colombia & 607938 & 11927 & 19364 & 380 & TRT \\
\hline México & 591712 & 4582 & 63819 & 494 & 620 \\
\hline España & 455621 & 9744 & 29011 & 587 & TRT \\
\hline Chile & 409974 & 21417 & 11244 & TRT
\end{tabular}

(607 938, TRT), México (591 712, TRT), España (455 621, TRT) y Chile (409 974, TRT).

El mayor número de muertes totales se observó en Estados Unidos (187 215, P), Brasil (120 896, P), India (64 617, U), México (63 819, TRT), Reino Unido (41 499, TRT), Italia (35 477, TRT), Francia (30 606, TRT), España (29 011, TRT), Perú (28 788, LP) e Irán (21 462, LP).

Los países con más casos por millón de habitantes fueron Qatar (42 230, LP), Bahrein (30 153, TRT), Chile (21 417, TRT), Panamá (21 284, LP), San Marino (20 917, A), Kuwait (19 772, TRT), Perú (19 584, LP), Aruba (18 692, sin dato de política), Estados Unidos (18 629, P), Brasil (18 149, P) y Omán (16 686, LP).

El mayor número de muertes por millón de habitantes se registró en San Marino (1237, A), Bélgica (853, TRT), Perú (871, LP), Andorra (686, LP), España (620, TRT), Reino Unido (611, TRT), Italia (587, TRT), Chile (587, TRT), Suecia (576, sin dato de política), Brasil $(568, \mathrm{P})$ y Estados Unidos $(565, \mathrm{P})$.

Los países con aplanamiento de la curva fueron Malasia (U), Haití (LP), Djibouti (LP), Tailandia (LP), Somalia (A), Sri Lanka (LP), Mali (LP), Estonia (R), Nueva Zelanda $(A)$, Uruguay (TRT), Chipre $(P)$, Georgia (TRT), Letonia (TRT), Burkina Faso (LP), Nigeria (sin dato de política), Andorra (LP), Vietnam (LP), San Marino (A), Taiwán (TRT), Mauricio (LP), Mongolia (LP), Camboya (P), Bután (R), Barbados (TRT), Brunei (P), Mónaco (TRT), Liechtenstein (R), Fiji (sin dato), Timor-Leste (TRT), Dominica (LP), Saint Kitts y Nevis (LP) y Ciudad del Vaticano (P).
Las Figuras 1 a 4 ilustran las diferencias en los países según sus políticas de uso de cubrebocas.

En la relación de la política de uso de mascarillas con el aplanamiento de la curva, no se encontró una asociación estadísticamente significativa ( $p=14718)$. La distribución de las variables cuantitativas tuvo un valor de $p=0.00$, por lo que se aplicó la prueba de Kruskal-Wallis. Las asociaciones de la política de uso de mascarilla con casos totales ( $p=0.01$ ), casos por millón $(p=0.04)$ y muertes por millón de habitantes $(p=0.02)$ resultaron estadísticamente significativas, no así con el número de muertes totales ( $p=0.222)$, Tabla 2. Las mismas variables tuvieron una asociación estadísticamente significativa $(p=0.00)$ con la tendencia de curva epidemiológica.

\section{Discusión}

Considerando la indiscutible relación entre el uso de mascarillas y un menor número de contagios, en la muestra analizada observamos que $15.81 \%$ de los países a nivel mundial no tenía alguna política establecida para el uso de cubrebocas (recomendado o ausente) y que entre los 10 primeros países con más casos, ninguno disponía de una política al respecto, si bien la convicción de adoptar el cubrebocas ha aumentado con el tiempo. ${ }^{14}$

Es notorio el bajo porcentaje global de países con una política universal $(5.08 \%)$ y una mayoría geográfica con indicaciones intermedias (casi $80 \%$ ). India llama la atención pues aparece en todas las listas de 
Tabla 2. Número de contagios y muertes en 129 países al 30 agosto de 2020, según la política de uso del cubrebocas

\begin{tabular}{|l|c|c|c|c|}
\hline Política & Total de contagios & Total de contagios/1000 000 habitantes & Muertes totales & Total de muertes/1000 000 habitantes \\
\hline U & 427951.5 & 1809.0 & 8466.7 & 60.8 \\
\hline TRT & 91612.1 & 5022.7 & 5586.8 & 161.5 \\
\hline P & 567213.9 & 3571.6 & 18816.0 & 96.7 \\
\hline R & 7728.8 & 2094.4 & 451 & 89.1 \\
\hline LP & 66210.7 & 4018.6 & 1926.2 & 80.0 \\
\hline A & 11342.7 & 3483.5 & 307.6 & 143.5 \\
\hline
\end{tabular}

$\mathrm{U}=$ universal, TRT = requerido en tiendas, restaurantes, transporte público; $\mathrm{P}=$ parcial, $\mathrm{R}=$ recomendado, $\mathrm{LP}=$ requerido en cualquier lugar público, $\mathrm{A}=$ ausente. Valor de $\mathrm{p}<0.05$ fue estadísticamente significativo.

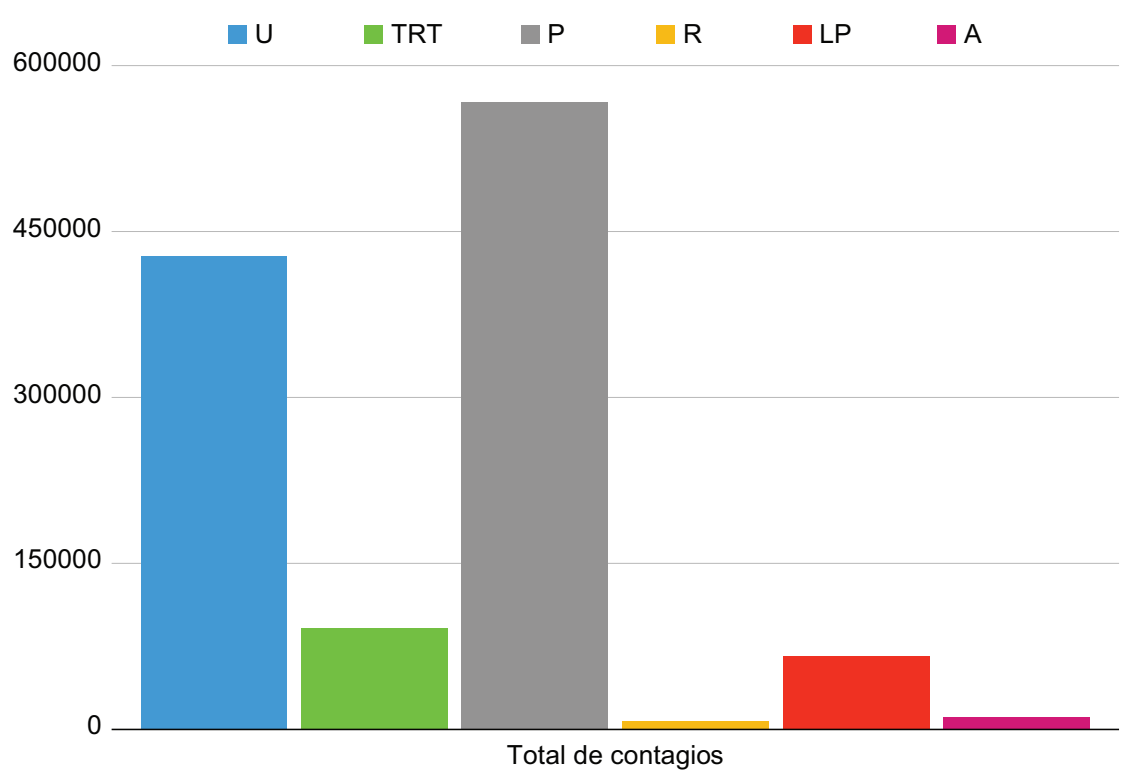

Figura 1. Diferencias entre las políticas de uso de mascarilla y los casos totales. $U=$ universal, $T R T=$ requerido en tiendas, restaurantes, transporte público; $P=$ parcial, $R=$ recomendado, $L P=$ requerido en cualquier lugar público, $A=$ ausente.

números elevados a pesar de la política de uso universal del cubrebocas. No obstante, la implementación tardía de esta pauta puede facilitar un número inicial acelerado de contagios. En el escenario de una exposición mucho más elevada, dado el número de enfermos con potencial de contagio, hay una mayor carga vírica en cada zona afectada. Una reciente publicación de The New England Journal of Medicine plantea que si la carga viral es importante para determinar la gravedad de la infección por el virus SARS-CoV-2, una razón hipotética para usar mascarillas sería la reducción del inóculo al que está expuesto el usuario y el impacto clínico posterior en la enfermedad. ${ }^{15}$
Tengamos en cuenta, además, el factor desinformación. ${ }^{16,17}$ Existen países en los cuales, a pesar de contar con la difusión de datos científicamente respaldados, una buena cantidad de sus habitantes se rehúsa a portar cubrebocas, bien sea por teorías de conspiración, reclamo de libertades individuales u otras ideas particulares propias de respuestas psicológicas frente a la crisis. ${ }^{18,19}$ Casos como los de las protestas realizadas en Estados Unidos 0 Alemania ${ }^{20,21}$ son los ejemplos más notorios, aunque es un hecho que México también se encuentra bajo la influencia de este tipo de fenómenos, con consecuencias como la violencia hacia el personal de salud. ${ }^{22}$ 


$6000 \quad \square \mathrm{U} \quad \square \mathrm{TRT} \quad \mathrm{P} \quad \square \mathrm{R} \quad \square \mathrm{LP} \quad \square \mathrm{A}$

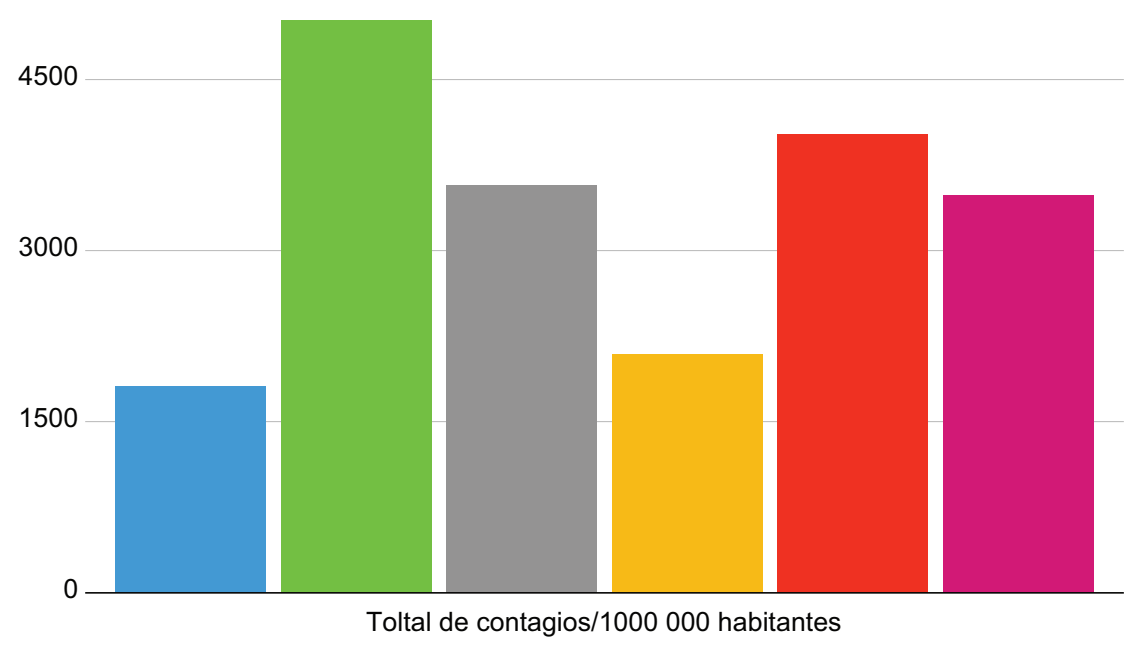

Figura 2. Diferencias entre las políticas de uso de mascarilla y los casos/1000 000 habitantes. $U=$ universal, $T R T=$ requerido en tiendas, restaurantes, transporte público; $P=$ parcial, $R=$ recomendado, $L P=$ requerido en cualquier lugar público, $A=$ ausente.

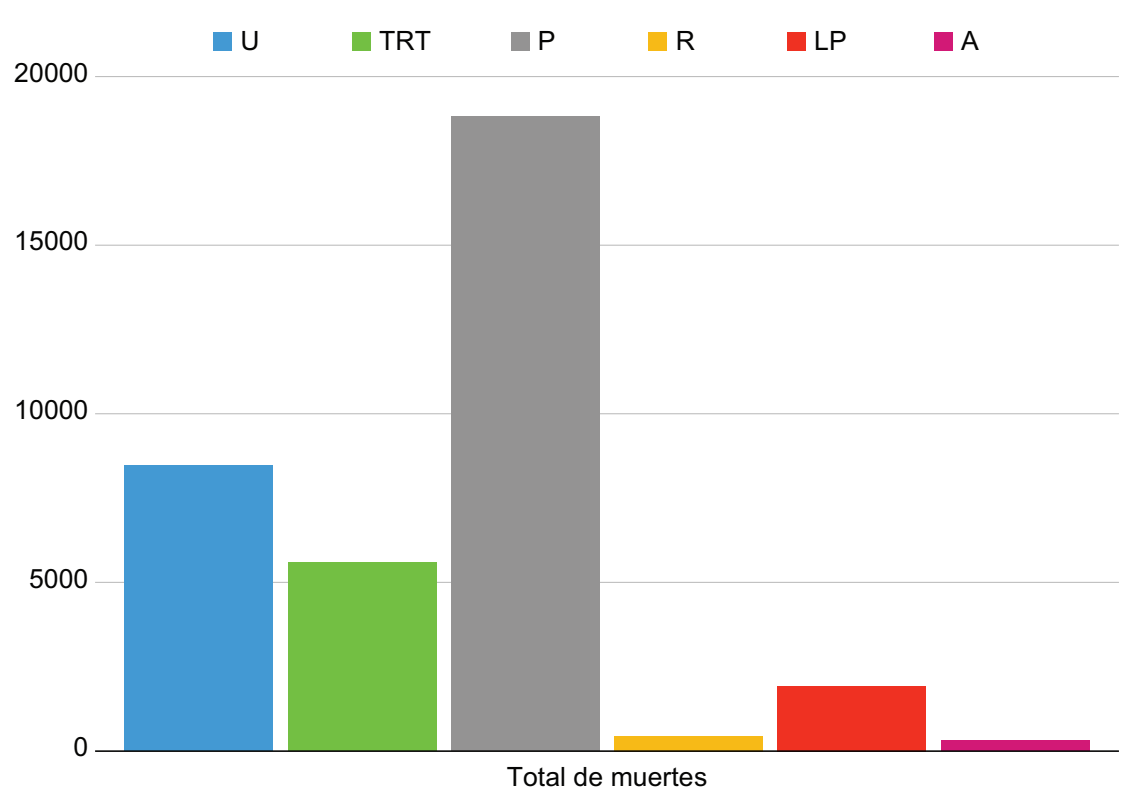

Figura 3. Diferencias entre las políticas de uso de mascarilla y las muertes totales. $U=$ universal, TRT = requerido en tiendas, restaurantes, transporte público; $P=$ parcial, $R=$ recomendado, $L P=$ requerido en cualquier lugar público, $A=$ ausente.

Por otro lado, se considera al distanciamiento social como el eje central de la disminución de contagios. ${ }^{13}$ Medidas complementarias como el lavado de manos, la protección ocular, la desinfección de superficies, el autocuidado ante síntomas de sospecha son parte de las múltiples recomendaciones de las que se tiene algún nivel de evidencia para su divulgación. . $^{13,23}$
Un elemento adicional tiene que ver con la reutilización de las mascarillas. La aplicación de una política obligatoria universal no necesariamente tiene en cuenta los recursos para su consecución y, menos aún, la posibilidad de renovación, además de que la medida también tiene un requerimiento básico de calidad. ${ }^{8} \mathrm{El}$ fenómeno de disparidad puede incluso ser considerado 


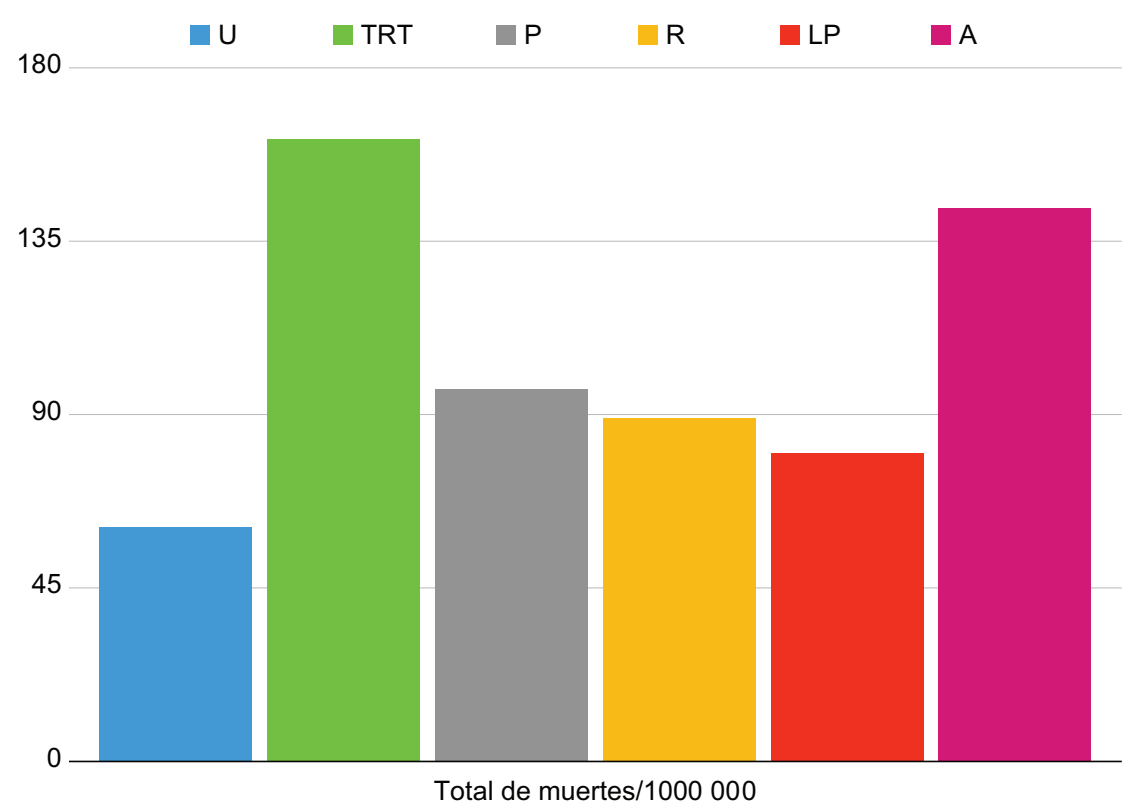

Figura 4. Diferencias entre las políticas de uso de mascarilla y las muertes por millón de habitantes. $U=$ universal, TRT = requerido en tiendas, restaurantes, transporte público, $P=$ parcial, $R=$ recomendado, $L P=$ requerido en cualquier lugar público, $A=$ ausente.

para entender las diferencias de conocimiento, actitud y prácticas de cuidado durante la pandemia. ${ }^{24}$

Consideremos también que las estadísticas proporcionadas ilustran el número de casos que se encuentran confirmados a través de las pruebas estandarizadas para este fin, por lo que las estimaciones que se postulan para corregir el subregistro podrían indicar un patrón diferente, ${ }^{25,26}$ estos números dependen de la cantidad y calidad de pruebas realizadas por cada país (por ejemplo, la sensibilidad de la reacción en cadena de la polimerasa [PCR] es casi de $100 \%$, pero su especificidad varía de 60 a $89 \%{ }^{27}$ ), del propio manejo de los datos contenidos en los registros e, incluso, del comportamiento regional de la transmisión del virus.

A pesar de lo descrito, es un hecho estadístico que en nuestra investigación encontramos una relación significativa entre la política de uso de mascarilla y el número de contagios, lo que respalda los hallazgos previos. ${ }^{13,23,28} \mathrm{El}$ análisis realizado hasta aquí es útil en la ausencia de un nexo estadísticamente significativo entre la política de uso de mascarilla y el fenómeno del aplanamiento de la curva.

La relación estadísticamente significativa entre la mortalidad/letalidad (en las muertes por millón de habitantes) y el uso de mascarillas, suma evidencia a las publicaciones que respaldan este impacto. ${ }^{28} \mathrm{Al}$ inicio se consideraba a la edad como el factor diferencial en la mortalidad; ${ }^{29}$ sin embargo, la evolución de la pandemia ha dado peso a las comorbilidades no solo respecto al riesgo de muerte, sino también en cuanto a la severidad del cuadro y la probabilidad de ser ingresado a una unidad de cuidado crítico. ${ }^{30}$ La obesidad, la hipertensión y la diabetes son los mayores factores de riesgo, ${ }^{23,31}$ así como males con los que la mayoría de los países con un alto índice de mortalidad están muy familiarizados por su alta prevalencia. Las características sociodemográficas, geográficas, ambientales y las enfermedades propias de cada región serán un elemento determinante para estas estimaciones. ${ }^{23}$ Si bien la política de uso de mascarillas no va a tener todo el peso en este impacto, no se puede pasar por alto que disminuir la velocidad de los contagios puede optimizar los recursos disponibles para la atención de los pacientes; esa fue la lección aprendida del paso de COVID-19 por Asia y Europa. ${ }^{32,33}$ Finalmente, hay que tener en cuenta que hasta la fecha no existe un tratamiento específico para esta enfermedad, hecho que obliga a proponer un modelo enfocado en la prevención. ${ }^{34}$

\section{Conclusiones}

La recomendación del uso generalizado de mascarilla es una medida con suficiente respaldo científico para reducir el número, velocidad de contagios y mortalidad por el virus SARS-Cov-2, por lo que es 
necesario considerarla al momento de establecer medidas de contención para episodios críticos similares al de la actual pandemia. El valor de una política generalizada es muy alto en el escenario de rebrote, con la necesidad de educación ciudadana. Existen múltiples variables adicionales que deben considerarse, las cuales se presentan más complejas al examinar el aspecto de la mortalidad/ letalidad.

Nuestras limitaciones en el análisis fueron la escasez de datos y que las fuentes no fueron las oficiales de cada país (aunque sí vinculadas a entidades académicas), así como el comportamiento dinámico de la información.

\section{Agradecimientos}

Agradecemos a los doctores Ramiro López Elizalde, Fiacro Jiménez Ponce, Juan Guadalupe Garza Cantú y Sandra Medina. De igual forma, a la Asociación Psiquiátrica Mexicana y al doctor Irving Vicente Gallardo Ramírez, por su apoyo y respaldo en el desarrollo de este trabajo. Por último a Jhons Hopkins University, al investigador Jeremy Howard, junto con todos los demás cofundadores y signatarios del movimiento \#Masks4All, y al profesor Yaneer Bar-Yam y todo su equipo de colaboradores en el movimiento EndCoronavirus.org, por generar, organizar, graficar y difundir de forma abierta datos que facilitaron la realización de este artículo.

\section{Conflicto de intereses}

Los autores declaran que no existe conflicto de intereses.

\section{Financiamiento}

Los autores declaran que no recibieron ninguna financiación o incentivo económico en la realización de este trabajo.

\section{Responsabilidades éticas}

Protección de personas y animales. Los autores declaran que para esta investigación no realizaron experimentos en seres humanos ni en animales.

Confidencialidad de los datos. Los autores declaran que en este artículo no aparecen datos de pacientes.
Derecho a la privacidad y consentimiento informado. Los autores declaran que en este artículo no aparecen datos de pacientes.

\section{Bibliografía}

1. Goh Y, Tan BYQ, Bhartendu C, Ong JJY, Sharma VK. The face mask: how a real protection becomes a psychological symbol during Covid-19? Brain Behav Immun. 2020;88:1-5.

2. Sanvicente-Vieira B, Kluwe-Schiavon B, Wearick-Silva LE, Piccoli GL, Scherer L, Tonelli HA, et al. Revised reading the mind in the eyes test (RMET) - Brazilian version. Rev Bras Psiquiatr. 2014;36:60-67.

3. Matuschek C, Moll F, Fangerau H, Fischer JC, Zänker K, van Griensven M, et al. The history and value of face masks. Eur J Med Res. 2020;25:23.

4. Brown CK. Respiratory protection against emerging infectious diseases: face masks, respirators, and tools for choosing between them. Health Secur. 2019:17:133-139.

5. Van der Sande M, Teunis P, Sabel R. Professional and home-made face masks reduce exposure to respiratory infections among the general population. PLoS One. 2008;3:e2618.

6. Davies A, Thompson KA, Giri K, Kafatos G, Walker J, Bennett A. Testing the efficacy of homemade masks: would they protect in an influenza pandemic? Disaster Med Public Health Prep. 2013;7:413-418.

7. Rengasamy S, Eimer B, Shaffer RE. Simple respiratory protection - Evaluation of the filtration performance of cloth masks and common fabric materials against 20-1000 nm size particles. Ann Occup Hyg. 2010:54:789-798.

8. Neupane BB, Mainali S, Sharma A, Giri B. Optical microscopic study of surface morphology and filtering efficiency of face masks. PeerJ. 2019:2019:e7142.

9. Feng S, Shen C, Xia N, Song W, Fan M, Cowling BJ. Rational use of face masks in the COVID-19 pandemic. Lancet Respir Med. 2020;8:434-436.

10. Javid B, Weekes MP, Matheson NJ. Covid-19: should the public wear face masks? BMJ. 2020;369:m1442.

11. Liu X, Zhang S. COVID-19: face masks and human-to-human transmission. Influenza Other Respir Viruses. 2020;14:472-473.

12. Organización Mundial de la Salud. Recomendaciones sobre el uso de mascarillas en el contexto de la COVID-19: orientaciones provisionales, 6 de abril de 2020. Suiza: Organización Mundial de la Salud; 2020.

13. Chu DK, Akl EA, Duda S, Solo K, Yaacoub S, Schünemann HJ, et al. Physical distancing, face masks, and eye protection to prevent person-to-person transmission of SARS-CoV-2 and COVID-19: a systematic review and meta-analysis. Lancet. 2020:395:1973-1987.

14. Quintana-Díaz MA, Aguilar-Salinas CA. Universal masking during COVID-19 pandemic. Current evidence and controversies. Rev Invest Clin. 2020;72:144-150.

15. Koven S. They call us and we go. N Engl J Med 2020; 382:1978-1979

16. Cuan-Baltazar JY, Muñoz-Pérez MJ, Robledo-Vega C, Pérez-Zepeda MF, Soto-Vega E. Misinformation of COVID-19 on the Internet: infodemiology study. JMIR Public Heal Surveill. 2020;6:e18444.

17. Aghagoli G, Siff EJ, Tillman AC, Feller ER. COVID-19: misinformation can kill. R I Med J. 2020;103:12-14

18. Fernández-Poncela A. Psicología de masas, identidad social, epidemias y rumores: la influenza en México. Sociológica. 2012;27:189-230.

19. Ho CS, Chee CY, Ho RC. Mental health strategies to combat the psychological impact of COVID-19 beyond paranoia and panic. Ann Acad Med Singapore. 2020;49:155-160.

20. BBC News [Internet]. Reino Unido: Coronavirus: US faced with protests amid pressure to reopen; 2020

21. BBC News [Internet]. Reino Unido: Coronavirus: thousands protest in Germany against restrictions; 2020.

22. Taylor L. Covid-19 misinformation sparks threats and violence against doctors in Latin America. BMJ. 2020;370.

23. Leffler CT, Ing EB, Lykins JD, Hogan MC, McKeown CA, Grzybowski A. Association of country-wide coronavirus mortality with demographics, testing, lockdowns, and public wearing of masks. Am J Trop Med Hyg. 2020;103:2400-2411.

24. Zhong BL, Luo W, Li HM, Zhang QQ, Liu XG, Li WT, et al. Knowledge, attitudes, and practices towards COVID-19 among chinese residents during the rapid rise period of the COVID-19 outbreak: a quick online cross-sectional survey. Int J Biol Sci. 2020;16:1745-1752.

25. Qin L, Sun $Q$, Wang $Y$, Wu KF, Chen M, Shia BC, et al. Prediction of number of cases of 2019 novel coronavirus (COVID-19) using social media search index. Int $\mathrm{J}$ Environ Res Public Health. 2020;17:2365.

26. Lauer SA, Grantz KH, Bi Q, Jones FK, Zheng Q, Meredith HR, et al. The incubation period of coronavirus disease 2019 (CoVID-19) from publicly reported confirmed cases: Estimation and application. Ann Intern Med. 2020;172:577-582. 
27. Yang Y, Yang M, Shen C, Wang F, Yuan J, Li J, et al. Evaluating the accuracy of different respiratory specimens in the laboratory diagnosis and monitoring the viral shedding of 2019-nCoV infections. 2020;1: 100061.

28. Eikenberry SE, Mancuso M, Iboi E, Phan T, Eikenberry K, Kuang Y, et al. To mask or not to mask: Modeling the potential for face mask use by the general public to curtail the COVID-19 pandemic. Infect Dis Model. 2020;5:293-308.

29. Kang SJ, Jung SI. Age-related morbidity and mortality among patients with COVID-19. Infect Chemother. 2020;52:154-164.

30. Jain V, Yuan JM. Predictive symptoms and comorbidities for severe COVID-19 and intensive care unit admission: a systematic review and meta-analysis. Int J Public Health. 2020;65:533-546.
31. Kumar A, Gupta R, Misra A. Comorbidities in COVID-19: outcomes in hypertensive cohort and controversies with renin angiotensin system blockers. Diabetes Metab Syndr Clin Res Rev. 2020;14:283-287.

32. Leung K, Wu JT, Liu D, Leung GM. First-wave COVID-19 transmissibility and severity in China outside Hubei after control measures, and second-wave scenario planning: a modelling impact assessment. Lancet. 2020;395:1382-1393.

33. Signorelli C, Scognamiglio T, Odone A. COVID-19 in Italy: impact of containment measures and prevalence estimates of infection in the general population. Acta Biomed. 2020;91:175-179.

34. Pascarella G, Strumia A, Piliego C, Bruno F, del Buono R, Costa F, et al. COVID-19 diagnosis and management: a comprehensive review. J Intern Med. 2020;288:192-206. 